\title{
The Influence of Nuclear Reactions and Nuclear Structure in Astrophysics
}

\author{
K. E. Rehm ${ }^{1, \star}$ \\ ${ }^{1}$ Physics Division, Argonne National Laboratory, Argonne, IL 60439, USA
}

\begin{abstract}
Nuclear reactions play an important role for the energy production and the nucleosynthesis in stars. New facilities, able to accelerate radioactive nuclei or high-intensity stable beams have allowed us to measure in the laboratory reactions involving short-lived nuclei or processes with very small cross sections, which are crucial for stellar nucleosynthesis. I will discuss some of the recent experiments studying fusion and transfer reactions with radioactive beams which play a critical role in various quiescent and explosive stellar environments.
\end{abstract}

\section{Introduction}

Nuclear structure and nuclear reactions involving the whole range from light to heavy nuclei are crucial for determining the format of our universe from its beginning to the present day. A well known example are the mass5 and mass- 8 stability gaps which restrict nucleosynthesis during the first few minutes of the Universe to the production of nuclei with $\mathrm{A} \leq 7$. Heavier nuclei are then 'cooked' in the high-density environment of stars or during stellar explosions. For this the nuclear shell structure causes the abundance peaks of certain elements around the socalled waiting-point nuclei in the vicinity of closed neutron shells.

The production of carbon, an element crucial for life on Earth, would not have been possible if Nature had not put an excited $0^{+}$level, the so-called Hoyle state, into the structure of ${ }^{12} \mathrm{C}$, with an energy located right in the Gamow window for the triple-alpha reaction.

Oxygen, another crucial element, which makes up about $2 / 3$ of the mass of the human body, and is, after hydrogen and helium the third-most abundant element in the universe, is produced in stars via the ${ }^{12} \mathrm{C}(\alpha, \gamma){ }^{16} \mathrm{O}$ reaction with a yield that is strongly affected by a subthreshold resonance in ${ }^{16} \mathrm{O}$. For the subsequent alpha capture reaction, ${ }^{16} \mathrm{O}(\alpha, \gamma){ }^{20} \mathrm{Ne}$, we find that the nuclear structure in ${ }^{20} \mathrm{Ne}$ provides no level with the proper quantum numbers, which inhibits this channel and restricts the destruction of ${ }^{16} \mathrm{O}$. These are just a few examples among the $6000 \mathrm{nu}-$ clei and 60,000 nuclear reactions participating in stellar nucleosynthesis showing the critical influence of nuclear structure and reactions in stellar environments.

In this contribution I will discuss three examples of this influence for three long-lived radio isotopes: ${ }^{60} \mathrm{Fe}$ $\left(\mathrm{T}_{1 / 2}=2.62 \mathrm{My}\right),{ }^{44} \mathrm{Ti}\left(\mathrm{T}_{1 / 2}=59 \mathrm{y}\right)$ and ${ }^{26} \mathrm{Al}\left(\mathrm{T}_{1 / 2}=0.717\right.$ $\mathrm{My}$. The fact that all of these isotopes have been de-

\footnotetext{
^e-mail: rehm@anl.gov
}

tected in our galaxy proves that nucleosynthesis is ongoing in stellar environments.

\section{$2{ }^{60} \mathrm{Fe}$ and the ${ }^{12} \mathrm{C}+{ }^{12} \mathrm{C}$ fusion reaction}

Among the three examples chosen for this presentation, ${ }^{60} \mathrm{Fe}$ is the isotope with the longest half-life: $\mathrm{T}_{1 / 2}=2.62 \pm$ 0.04 My [1, 2], which, however, is still short compared to the age of the solar system $(\sim 4.6 \mathrm{By}) .{ }^{60} \mathrm{Fe}$ material has been found in the interstellar medium through $\gamma$-ray measurements with the INTEGRAL satellite, in cosmic rays with the CRIS detector aboard the Advanced Composition Explorer (ACE) Spacecraft [3] and on Earth in deep-sea sediments, which are thought to originate from recent ( 2.2 My) and close (60-130 parsecs) supernovae explosions $[4,5]$. Since ${ }^{60} \mathrm{Fe}$ is located on the neutron-rich side of the mass valley its production requires a complex reaction path which is discussed in more detail in Ref. [6]. Although the nuclei produced in the fusion of $\mathrm{C}+\mathrm{C}$ are far away in mass and $\mathrm{Z}$ from ${ }^{60} \mathrm{Fe}$, this reaction still plays a critical role for the production of ${ }^{60} \mathrm{Fe}$.

Fusion of ${ }^{12} \mathrm{C}+{ }^{12} \mathrm{C}$ has been studied extensively over the years down to the nb region. The measurements at the lowest energies are quite challenging due to the small yields and the strong influence from various background reactions. For that reason, extrapolation techniques have been developed in order to obtain cross sections at energies where no experimental data are available. This extrapolation became more complicated when fusion hindrance at sub-barrier energies was discovered in 2002 [7]. As discussed in Ref. [8], fusion reactions involving mediummass nuclei with negative $\mathrm{Q}$-values require a maximum in the astrophysical S-factor $(S(E)=\sigma E \exp (2 \pi \eta)$ where $\eta=Z_{1} Z_{2} e^{2} /(\hbar v)$ is the Sommerfeld parameter $)$, since the fusion cross section has to reach zero at a c.m. energy of $\mathrm{E}=-\mathrm{Q}$. For fusion reactions with positive $\mathrm{Q}-\mathrm{values}$, this occurs at unphysical (negative) c.m. energies and, thus, no maximumof the $\mathrm{S}$-factor is required. However, several re- 
cent experiments involving lighter nuclei with positive Qvalues have shown that an $\mathrm{S}$-factor maximum is observed for these systems as well [9].

In order to study the fusion hindrance in the ${ }^{12} \mathrm{C}+{ }^{12} \mathrm{C}$ system, we have recently measured $\mathrm{C}+\mathrm{C}$ fusion cross sections using the particle- $\gamma$-coincidence technique [10]. Details of the detection technique can be found at other places in these proceedings [11]. The resulting $\mathrm{C}+\mathrm{C} \mathrm{S}$-factors from this and two other recent experiments are shown in Fig. 1.

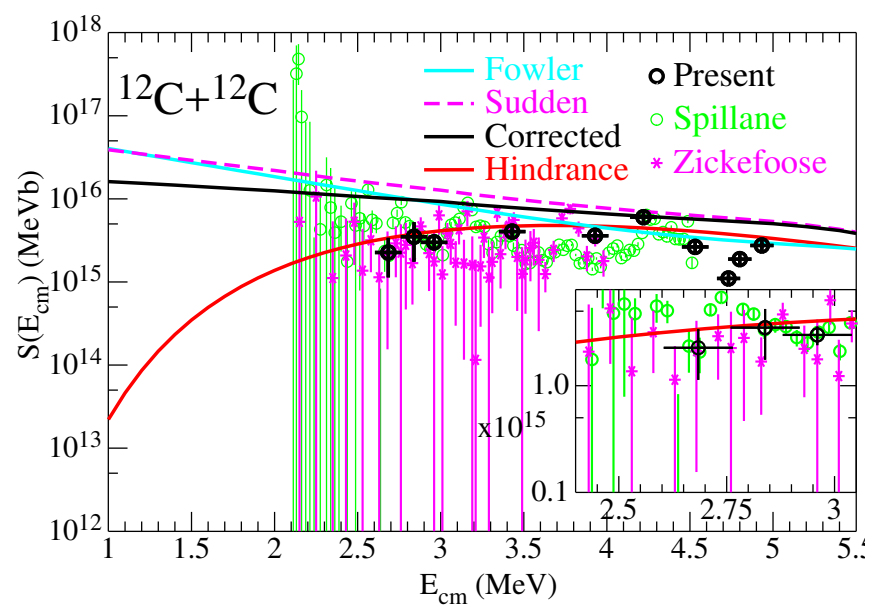

Figure 1. S-factors for ${ }^{12} \mathrm{C}+{ }^{12} \mathrm{C}$ fusion from the particle- $\gamma$ measurement (black points) in comparison to the results from recent $\gamma$ (green) or particle (magenta) experiments, respectively $([12,13])$. The lines are S-factor extrapolations from Refs. [1417].

The predictions of various extrapolation results are given by the solid and dashed lines. While most of these extrapolations increase towards lower energies, the inclusion of fusion hindrance (see red solid line) predicts a maximum of the $\mathrm{S}$-factor at about $\mathrm{E}_{\text {c.m. }}=3.5 \mathrm{MeV}$, in agreement with the solid black points from the particle- $\gamma$ experiment.

This S-factor maximum and the resulting reduction of the fusion cross sections has important consequences for the production of ${ }^{60} \mathrm{Fe}$. As discussed in Ref. [6], hindrance of carbon fusion in stellar environments will delay the carbon ignitition by shifting it to higher densities and temperatures. This leads to a higher neutron production and, thus, to an increased ${ }^{60} \mathrm{Fe}$ (as well as ${ }^{26} \mathrm{Al}$ ) production yield. In Ref. [18] a comparison of the ${ }^{60} \mathrm{Fe}$ yield extracted from Accelerator Mass Spectrometry measurements of deep-sea sediments and predictions from a variety of supernovae models has been provided. An increase in the ${ }^{60} \mathrm{Fe}$ production cross section will strongly influence the conclusions regarding the age and distance of the supernovae explosions. For that reason more high-quality fusion cross sections for ${ }^{12} \mathrm{C}+{ }^{12} \mathrm{C}$ at even lower energies are clearly needed.

\section{Reactions producing and destroying ${ }^{44} \mathrm{Ti}$}

The relatively short half-life of $59.0 \pm 0.6$ y restricts the use of this radioisotope to more recent stellar events and for that reason so far only two sites are known where ${ }^{44} \mathrm{Ti}$ has been detected. One is the supernovae remnant Cassiopeia A, which was seen on Earth about 350 years ago and is located at a distance of $\sim 3.4$ kparsecs. The other is the well-studied recent supernova 1987A in the neighboring Large Magellanic Cloud at a distance of 51.4 kparsecs. First seen by the COMPTEL and INTEGRAL satellites $[19,20]$, recent measurements by the NUSTAR satellite [21] have provided data with unprecedented position resolution to resolve the globular distribution of ${ }^{44} \mathrm{Ti}$ from the SN explosion.

The isotope ${ }^{44} \mathrm{Ti}$ is produced through the ${ }^{40} \mathrm{Ca}(\alpha, \gamma){ }^{44} \mathrm{Ti}$ reaction in the alpha-rich freeze-out phase of the expanding supernova shock front, but it can be destroyed again via the ${ }^{44} \mathrm{Ti}(\alpha, \mathrm{p})^{47} \mathrm{~V}$ process. Sensitivity studies have provided a list of reactions that are critical for interpreting the abundance measurements from the satellite measurements $[22,23]$.

For the production reaction, ${ }^{40} \mathrm{Ca}(\alpha, \gamma){ }^{44} \mathrm{Ti}$, several experiments have been performed at various laboratories during the last 10 years using a variety of detection techniques. The reaction rates from these measurements were found to agree with each other within a factor of about 1.5 [24], but were by a factor of 3 larger than the rates from an earlier measurement [25]. This increased production rate is in agreement with the deduced amounts of ${ }^{44} \mathrm{Ti}$ in the two supernovae sites mentioned above. This comparison, however, also depends on processes that can destroy ${ }^{44} \mathrm{Ti}$, e.g. via the ${ }^{44} \mathrm{Ti}(\alpha, \mathrm{p}){ }^{47} \mathrm{~V}$ reaction. Since a measurement of this reaction requires the use of a radioactive ${ }^{44} \mathrm{Ti}$ beam, only two experiments have been performed so far. In the first measurement [26], the heavy ${ }^{47} \mathrm{~V}$ recoil nuclei from the inverse ${ }^{4} \mathrm{He}\left({ }^{44} \mathrm{Ti},{ }^{47} \mathrm{~V}\right) \mathrm{p}$ reaction were detected and identified according to mass, $\mathrm{Z}$ and energy in the focal plane of a recoil separator. While this approach allows us to separate contributions from stable beam contaminants (e.g. ${ }^{44} \mathrm{Ca}$ ), the detection efficiency (determined by the charge-state selection in the recoil separator) allows measurements down to about $1 \mathrm{mb}$ using present-day radioactive beam intensities. In a more recent experiment [27], the outgoing protons were detected in an array of position-sensitive Si detectors. In this experiment, an upper limit of the energy-integrated cross section of about 60 $\mu \mathrm{b}$ was determined. This technique, however, suffers from the same difficulties encountered in the ${ }^{12} \mathrm{C}+{ }^{12} \mathrm{C}$ fusion experiments, i.e. a sensitivity to background protons produced e.g. in the $\mathrm{Al}$ windows of the helium gas target.

In order to extend these measurements to lower energies we have developed a new high-efficiency detector system which is based on the active target technique [28]. A cross section of the gridded multi-sampling ionization chamber (MUSIC) is shown in Fig. 2a. The anode of the ionization chamber consists of 16 strips, which are partitioned into right and left sections. The two additional strips at the entrance and exit of the detector are used for particle identification. The ionization chamber is filled with helium gas, which acts as both target and detection gas.

The incident beam passing through the detector produces a certain amount of electrons in each individual an- 
ode strip. If at a given location along the particle trajectory inside the detector an $(\alpha, \mathrm{p})$ reaction occurs, the recoil products, which have a higher nuclear charge, will produce a larger energy loss signal. Since the $(\alpha, \mathrm{p})$ reaction is studied in inverse kinematics, an angle- and energy-integrated cross section is determined in the experiment. Furthermore, this technique is 'self-normalizing' since reaction products as well as the incident beam particles are measured by the same detector. Simulations and first measurements of $(\alpha, \mathrm{p})$ (as well as $(\alpha, \mathrm{n}))$ reactions with ${ }^{17} \mathrm{O}$ and ${ }^{23} \mathrm{Na}$ beams have already been performed [29]. Based on these results we have made Monte Carlo simulations about the expected response of the detector for the ${ }^{44} \mathrm{Ti}(\alpha, \mathrm{p})^{47} \mathrm{~V}$ reaction, which are shown in Fig. 3. First experiments with ${ }^{44} \mathrm{Ti}$ are planned as soon as beams from the new AIRIS separator [30] at ATLAS will become available.
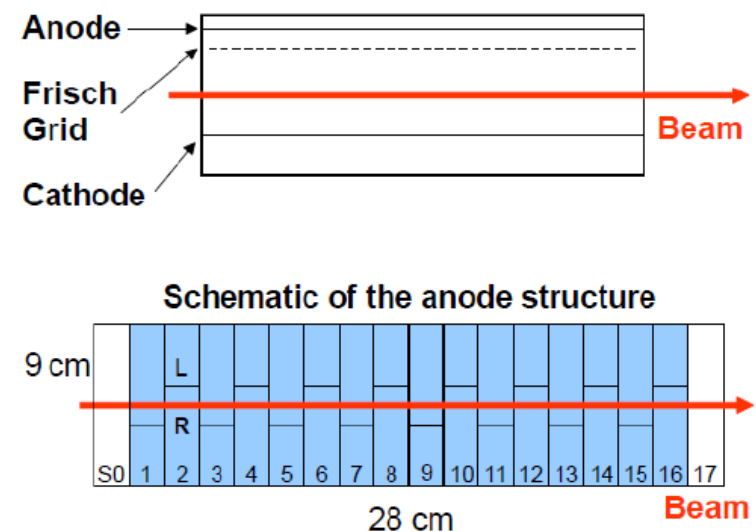

Figure 2. Schematic of the Multi-Sampling Ionization Chamber MUSIC.

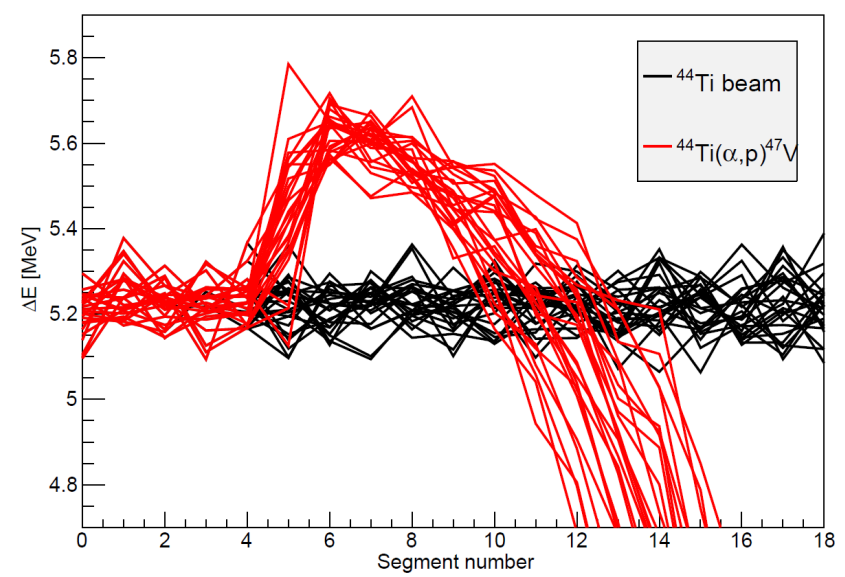

Figure 3. Monte Carlo simulation of the signals from the ${ }^{44} \mathrm{Ti}$ beam and the ${ }^{47} \mathrm{~V}$ recoils resulting from the ${ }^{4} \mathrm{He}\left({ }^{44} \mathrm{Ti},{ }^{47} \mathrm{~V}\right) \mathrm{p}$ reaction.

\section{Production of an isomeric ${ }^{26} \mathrm{Al}\left(0^{+}\right)$beam with the in-flight technique}

Among the three radioisotopes discussed in this presentation, ${ }^{26} \mathrm{Al}$ is perhaps the most well studied example. The detection of the characteristic $1.809-\mathrm{MeV} \gamma$ ray line from the decay of the long-lived radioisotope ${ }^{26} \mathrm{Al}\left(\mathrm{T}_{1 / 2}=7.17 \times 10^{5} \mathrm{y}\right)$ in the interstellar medium by the COMPTEL and INTEGRAL $\gamma$-ray satellites [31, 32] has demonstrated that nucleosynthesis is still ongoing in the Galaxy. From the detailed maps of the ${ }^{26} \mathrm{Al}$ distribution, an equilibrium mass of $\sim 2-3$ solar masses of ${ }^{26} \mathrm{Al}$ in the Galaxy [32] has been deduced. ${ }^{26} \mathrm{Al}$ can be produced in a number of different sources: in massive Wolf-Rayet (WR) stars either during the hydrogen-burning or the core collapse supernova (CCSN) phase [32], with additional contributions from asymptotic giant branch (AGB) stars and classical novae $(\mathrm{CN})[33,34]$. A detailed understanding of the production mechanism, however, is complicated by the existence of a short-lived $\left(\mathrm{T}_{1 / 2}=6.4 \mathrm{~s}\right)$ isomeric $0^{+}$state in ${ }^{26} \mathrm{Al}$, located $228-\mathrm{keV}$ above the $J^{\pi}=5^{+}$ground state. This state decays by $\beta^{+}$emission and is therefore 'invisible' to $\gamma$-ray satellites. While the communication between the ground state and the isomeric state is inhibited by the large spin difference $(\Delta \mathrm{J}=5)$, the two states may communicate with each other via thermal excitations involving higher-lying levels in ${ }^{26} \mathrm{Al}$ which puts constraints on the production and destruction processes of ${ }^{26} \mathrm{Al}$ in astrophysical environments.

Two experiments of the ${ }^{26} \mathrm{Al}\left(5^{+}\right)(\mathrm{d}, \mathrm{p})^{27} \mathrm{Al}$ reaction were conducted recently with the goal to extract spectroscopic information of key resonances in ${ }^{27} \mathrm{Si}$ via its mirror symmetry. From these results the ${ }^{26} \mathrm{Al}\left(5^{+}\right)(\mathrm{p}, \gamma)^{27} \mathrm{Si}$ reaction rate in WR stars and AGB stars is well constrained $[27,35]$. For the isomeric state, however, estimates of the ${ }^{26} \mathrm{Al}\left(0^{+}\right)(\mathrm{p}, \gamma)^{27} \mathrm{Si}$ reaction rate are presently based on Hauser-Feshbach calculations by scaling the ground state $(\mathrm{p}, \gamma)$ rate [37].

Several attempts have been made at different laboratories worldwide to produce an isomeric ${ }^{26} \mathrm{Al}\left(0^{+}\right)$beam with sufficient intensity, purity, energy resolution and a high isomer-to-ground state ratio in order to study nuclear reactions induced by the $0^{+}$isomer in ${ }^{26} \mathrm{Al}$ [38].

At the ATLAS accelerator at Argonne National Laboratory we have recently produced an ${ }^{26} \mathrm{Al}\left(0^{+}\right)$ beam with $70 \%$ isomeric content and performed a first ${ }^{26} \mathrm{Al}\left(0^{+}\right)(\mathrm{d}, \mathrm{p}){ }^{27} \mathrm{Al}$ experiment [38]. The ${ }^{26} \mathrm{Al}\left(0^{+}\right)$isomer was produced via the inverse $(\mathrm{p}, \mathrm{n})$ reaction $\mathrm{p}\left({ }^{26} \mathrm{Mg},{ }^{26} \mathrm{Al}\right) \mathrm{n}$. For that a $6.1 \mathrm{MeV} / \mathrm{u}$ primary beam of ${ }^{26} \mathrm{Mg}$ bombarded a gas cell filled with $1 \mathrm{~atm}$ of hydrogen gas. The energy of the primary beam and the target pressure in the gas cell were chosen to maximize the production of the isomeric state over the ground state. The isomeric ${ }^{26} \mathrm{Al}^{13+}$ beam was separated from the $12^{+}$charge state of the ${ }^{26} \mathrm{Mg}$ production beam through its different magnetic rigidity using a bending magnet in the beam line system [39].

In a first step, a test experiment was performed using a rotating stopper setup to measure the purity and isomer content of the beam. The radioactive ${ }^{26} \mathrm{Al}$ beam was stopped in a $100 \mathrm{mg} / \mathrm{cm}^{2}$ thick Au-catcher foil mounted 
on a rotating wheel. After an implantation time of $15 \mathrm{sec}$ the foil was rotated by $180^{\circ}$ and placed in between two face-to-face $\mathrm{NaI}$ detectors where the two coincident 511$\mathrm{keV}$ photons from the $\beta^{+}$decay of ${ }^{26} \mathrm{Al}\left(0^{+}\right)$were detected. The measured half-life of $6 \pm 1 \mathrm{sec}$ confirmed the isomeric content of the beam. During the $\gamma$-counting time the full ${ }^{26} \mathrm{Al}$ beam was transmitted to the Split-Pole Spectrograph where the beam was identified with respect to mass, $\mathrm{Z}$ and its total energy. GEANT4 simulations were performed to calculate the detection efficiencies of the $\mathrm{NaI}$ detectors.

The purity of the ${ }^{26} \mathrm{Al}$ beam was better than $98 \%$ as shown in Fig. 4a in a spectrum measured with a silicon surface barrier detector at $0^{\circ}$. Fig. $4 \mathrm{~b}$ shows the coincident 511-keV annihilation radiation measured with two $\mathrm{NaI}$ detectors. The inset confirms the presence of $0^{+}$isomer through its known $6.35 \mathrm{~s}$ half-life. From this beam development run it was established that, for this specific production energy and target thickness, $70 \pm 10 \%$ of the radioactive ${ }^{26} \mathrm{Al}$ beam was in the isomeric $0^{+}$state. The main contaminant in the ${ }^{26} \mathrm{Al}^{13+}$ beam came from the primary ${ }^{26} \mathrm{Mg}^{11+}$ production beam with an energy of $86 \mathrm{MeV}$ while the ${ }^{26} \mathrm{Mg}^{12+}$ charge state was completely eliminated by a RF sweeper. Details about the experiment studying the ${ }^{26} \mathrm{Al}\left(0^{+}\right)(\mathrm{d}, \mathrm{p})^{27} \mathrm{Al}$ reaction can be found in Ref. [38].

\section{Summary}

The examples presented in this contribution are a clear indication of the close relationship that exists between observational astronomy, nuclear physics and computational astrophysics. New developments in these fields will improve our knowledge of stellar events over the next few years. New Earth and space-based observatories, covering all wavelengths, cutting-edge facilities to accelerate high-intensity stable and radioactive beams and new and faster computers will provide the framework to keep astrophysics in the forefront of science.

\section{Acknowledgements}

The author wants to thank C. L. Jiang and the collaborators from the ${ }^{12} \mathrm{C}+{ }^{12} \mathrm{C}$ fusion experiment as well as $\mathrm{S}$. Almaraz-Calderon and the collaborators from the ${ }^{26} \mathrm{Al}\left(0^{+}\right)$ experiment for permission to present results before publication. I also want to thank M. Avila and R. Talwar from the ANL MUSIC collaboration for some of the data from the active target system. This work was supported by the US Department of Energy, Office of Nuclear Physics, under Contract No. DEAC02-06CH11357. This research used resources of ANL's ATLAS facility, which is a DoE Office of Science User Facility.

\section{References}

[1] G. Rugel et al., Phys. Rev. Lett. 103, 072502 (2009).

[2] A. Wallner et al., Phys. Rev. Lett. 114, 041101 (2015).

[3] W. R. Binns et al., Science 352, 677 (2016).

[4] K. Knie et al., Phys. Rev. Lett. 93, 171103 (2004).

[5] A. Wallner et al., Nature 532, 69 (2016).
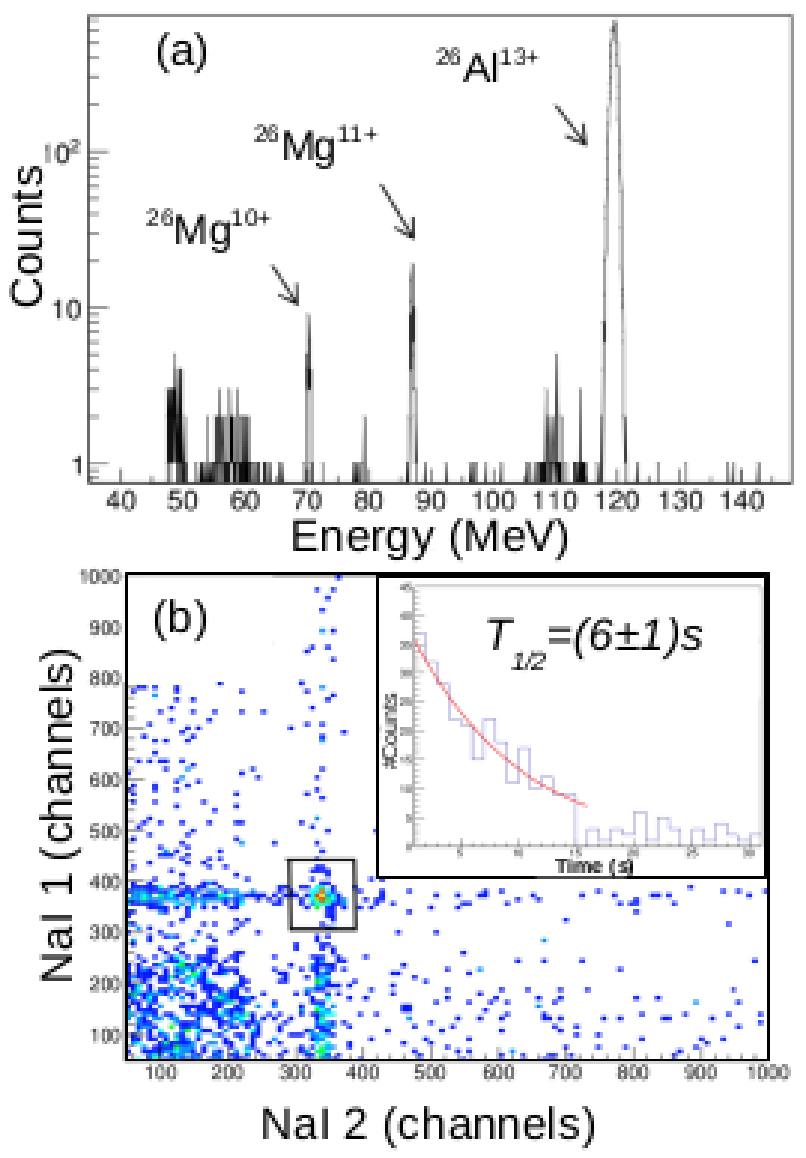

Figure 4. a) Energy spectrum of the secondary ${ }^{26} \mathrm{Al}^{13+}$ beam measured in a Si detector. The main beam contaminant originates from the energy-degraded ${ }^{26} \mathrm{Mg}^{11^{+}}$beam. b) $\gamma-\gamma$ coincidence spectrum measured with two NaI detectors. The insert shows the time dependence confirming the production of a $6.35 \mathrm{~s}{ }^{26} \mathrm{Al}\left(0^{+}\right)$ beam.

[6] L. R. Gasques et al., Phys. Rev. C 76, 035802 (2007).

[7] C. L. Jiang et al., Phys. Rev. Lett. 89, 052701 (2002).

[8] C. L. Jiang et al., Phys. Rev. C 73, 014613 (2006).

[9] C. L. Jiang et al., to be published.

[10] C. L. Jiang et al., Nucl. Instrum. Meth. in Phys. Res. A 682, 12 (2012).

[11] S. Courtin, Proceedings of this conference.

[12] T. Spillane et al., Phys. Rev. Lett. 98, 122501 (2007).

[13] J. Zickefoose, Thesis, Univ. of Connecticut (2011), unpublished.

[14] W. Fowler, G. Caughlan and B. Zimmerman, Ann. Rev. Astrophys. 13, 69 (1975).

[15] H. Esbensen, X. D. Tang and C. L. Jiang, Phys. Rev. C 84, 064613 (2011).

[16] C. L. Jiang et al., Phys. Rev. Lett. 110, 072701 (2013).

[17] C. L. Jiang et al., Phys. Rev. C 75, 015803 (2007).

[18] D. Breitschwerdt et al., Nature 532, 73 (2016).

[19] A. F. Iyudin et al., Astron. Astrophys. 284, L1 (1994).

[20] S. A. Grebenev et al., Nature 490, 373 (2012). 
[21] B. W. Grefenstette et al., Nature 506, 339 (2014).

[22] L. S. The, D. D. Clayton, L. Jin and B. S. Mayer, Astrophys. J 504, 500 (1998).

[23] G. Magkotsios et al., Astrophys. J. Suppl. Ser. 191, 66 (2010).

[24] D. Robertson et al., Phys. Rev. C 85, 045810 (2012) and references therein.

[25] E. L. Cooperman, M. H. Shapiro and H. Winkler, Nucl. Phys. A 284, 163 (1977).

[26] A. A. Sonzogni et al., Phys. Rev. Lett. 84, 1651 (2000).

[27] V. Margerin et al., Phys. Lett. B 731, 358 (2014).

[28] P. F. F. Carnelli et al., Nucl. Instrum. Meth. in Phys. Res. A 799, 197 (2015).

[29] M. L. Avila et al., Nucl. Instrum. Meth. in Phys. Res. A 859, 63 (2017).
[30] B. B. Back, Proceedings of this conference.

[31] R. Diehl et al., Astron. Astrophys. 298, 445 (1995).

[32] R. Diehl et al., Nature 439, 45 (2006).

[33] L. Siess and M. Arnould, Astron. Astrophys. 489, 395 (2008).

[34] J. Jose, M. Hernanz and A.Coc, Astrophys. J. 479, L55 (1997).

[35] S. Pain et al., Phys. Rev. Lett. 114, 212501 (2015).

[36] V. Margerin et al., Phys. Rev. Lett. 115, 062701 (2015).

[37] C. Iliadis, A. Champagne, A. Chieffi and M. Limongi, Astrophys. J. Suppl. Ser. 193, 16 (2011).

[38] S. Almaraz-Calderon et al., to be published.

[39] B. Harss et al., Rev. Sci. Instrum. 71, 380 (2000). 